\title{
Fault Detection for a Class of Uncertain Linear Systems
}

\author{
Qingyu Su and Jian Li \\ School of Automation Engineering, Northeast Dianli University, Jilin 132012, China \\ Correspondence should be addressed to Qingyu Su; suqingyuphd@yeah.net
}

Received 3 July 2013; Accepted 15 August 2013

Academic Editor: Bochao Zheng

Copyright (C) 2013 Q. Su and J. Li. This is an open access article distributed under the Creative Commons Attribution License, which permits unrestricted use, distribution, and reproduction in any medium, provided the original work is properly cited.

\begin{abstract}
Design problem of the fault detection filter for a class of uncertain feedback systems is discussed in this paper. The system under consideration is the model with the nonstandard parameter uncertain regions. The fault detection filter design problem is reduced to an optimized filter design problem, which maximizes the sensitivity of the fault in the fault cases and meets the disturbance attenuation performance in both fault-free cases and fault cases, simultaneously. A numerical example is used to demonstrate the effectiveness of the proposed design method.
\end{abstract}

\section{Introduction}

In recent years, fault detection technique is given more and more attentions for the higher demands of safety and reliability of the control systems. A considerable sum of results on fault detection, both in the light of the datadriven and the model-based detection techniques, have been achieved (see [1-3]). Among the model-based techniques, a number of results which depend on the linear matrix inequality (LMI) techniques have appeared to deal with the fault detection problem. References [4-6] investigate the estimation problem of the fault by designing a filter. Recently, a so-called $H_{\infty} / H_{-}$design approach for the fault detection problem has received considerable attentions. Further, the fault detection problem associated with some certain $H_{\infty} / H_{-}$ performance indices is discussed in [7-10]. The fault models considered in the above papers are classical and can describe a large class of the fault types. However, it should be pointed out that the actuator stuck fault also needs to be investigated when the amplitude of the faults are not larger enough to be detected.

At the same time, most of the models are obtained by the identification experiments with some limited input/output data. Identification experiment in the prediction error (PE) framework delivers an uncertain region which contains the true system at some (user-chosen) probability level [11]. The uncertain region can result in an identified parametric ellipsoid set which is regarded as a nonstandard parameter uncertain structure in the related classical papers. The ellipsoidal uncertain structure is studied by some results; see [12-15] which reduce the uncertain region by employing the robust tools. Compared with the stability and the stabilization problems for this particular uncertainty, there are no results that to pay attention to deal with the fault detection problem with the models which have the ellipsoidal uncertainty. Moreover, since the existing approaches about fault detection (FD) are not appropriate for FD problem for this kind of uncertainty, the new FD technique should solve this case.

Motivated by the above reasons, the optimization design problem of the FD filter for uncertain linear feedback system is studied. The design objective is to maximize the sensitivity of the fault in the fault cases, which subjects to the disturbances attenuation performance in both fault-free cases and the fault cases. In practical, the frequency of the disturbance signals and the fault signals are usually in bound. Thus, a notion of the finite-frequency performance indices is introduced to describe the performance indices; LMIs-based sufficient conditions are provided using the finite-frequency approach proposed in $[16,17]$. The optimal solution of gain matrices of the FD filter can be obtained.

The main contribution of this paper that the direct design approach of the FD filter proposed to the linear model with elliptical uncertain structure. Unlike other fault detection approaches for the uncertain model, in which the FD filter is achieved not only depending on the known 
lower and known upper bounds of the uncertainty but also extracting convex polygons of the uncertain region, a general parametrization for the set of multipliers is introduced to decrease the conservatism. To the best of our knowledge, such a framework for the fault detection with the elliptical parameter uncertainty structure has not been reported in the literature.

The paper is organized as follows. Section 2 introduces the problem under consideration and presents the design objectives. Section 3 illustrates a FD filter design approach in detail. The algorithm is given in Section 4. An illustrative example is given in Section 5 to demonstrate the proposed method. Conclusions to this work are given in the last section.

Notation. For a matrix $A, A^{T}$ denotes its transpose. For a symmetric matrix, $A>0(A \geq 0)$ and $A<0(A \leq$ $0)$ denote positive-definiteness (positive semidefinite matrix) and negative-definiteness (negative semi-definite matrix), respectively. The Hermitian part of a square matrix $M$ is denoted by $\operatorname{He}(M):=M+M^{T}$. The symbol $*$ within a matrix represents the symmetric entries. $\sigma_{\min }(G)$ denotes minimum singular values of the transfer matrix $G$. The symbol $\otimes$ denotes the Kronecker product, and 0 denotes zero matrix with appropriate dimension. Both $I$ and $\mathscr{I}$ denote identity matrix with appropriate dimension. $\mathscr{I}_{0}$ and $\mathscr{I}_{1}$ denote the matrices $[10 ; 00]$ and $[1 ; 0]$ of appropriate dimensions, respectively.

\section{Problem Formulation}

2.1. System Model. Consider the following uncertain linear systems:

$$
\begin{gathered}
\dot{x}(t)=A x(t)+B_{1} d(t)+B_{2} f(t)+L_{1} w(t), \\
y(t)=C x(t)+D_{1} d(t)+D_{2} f(t)+L_{2} w(t), \\
w(t)=\Delta z(t), \\
z(t)=H x(t) \quad \Delta \in \Delta,
\end{gathered}
$$

where $x(t)$ is the state $y(t)$ is the output, $u(t)$ is the input, and $d(t)$ is an energy-bounded disturbance; $r_{0}$ is the reference input, $x(t)$ is the state; $A, B_{1}, B_{u}, C, D, L_{1}, L_{2}$, and $H$ are known constant matrices of appropriate dimensions. Consider that $\Delta \in \Delta$, where $\Delta$ is defined as

$$
\Delta=\operatorname{diag}(\underbrace{\theta, \ldots, \theta}_{n_{0}}), \quad \theta=\left[\theta_{1}^{T}, \ldots, \theta_{n_{\theta}}^{T}\right]^{T} \in R^{n_{\theta}},
$$

where $n_{0}$ is the number of $\theta$ in $\Delta$, then the dimension of the $\Delta$ is $n_{\theta} n_{0} \times n_{0}$, and $\Delta$ is the parameter set of $\Delta$. The uncertain parameter $\theta$ with a certain use-chosen probability $(\mathscr{X})$ is at the uncertain set $\mathcal{U}$. The uncertain set $\mathcal{U}$ is an ellipsoid in the parameter space:

$$
\mathcal{U}=\left\{\theta \mid \theta^{T} \mathscr{R} \theta<\mathscr{X}\right\},
$$

where $\mathscr{R}$ describes the form of the ellipsoid and $\mathscr{X}$ is a constant computed by the desired probability in model identification.
Remark 1. The parameter uncertainty region in (1) is ellipsoidal. This kind of particular uncertainty is derived from classical prediction error identification, and it is a typical parameter uncertainty of the model which is identified. Although the parameter uncertainty can turn into the polytopic uncertainty by extracting convex polygons of the ellipsoidal region, this transformation method enlarges the the uncertain region and increases the conservatism. In this paper, the ellipsoidal uncertainty is disposed directly.

To detect the actuator stuck faults, the FD filter is designed such that the residual $r(t)$ can be obtained as

$$
\begin{gathered}
\dot{x}_{f}(t)=A_{f} x_{f}(t)+B_{f} y(t), \\
r(t)=C_{f} x_{f}(t)+D_{f} y(t),
\end{gathered}
$$

where $x_{f}$ is the state of the FD filter. Consider that $A_{f} \in$ $R^{n_{f} \times n_{f}}, B_{f} \in R^{n_{f} \times n_{m}}, C_{f} \in R^{n_{f}}, D_{f} \in R^{m}$.

By combining (1) and (4), we have the following augmented systems:

$$
\begin{gathered}
\xi(t)=\bar{A} \xi(t)+\bar{B}_{d} d(t)+\bar{L}_{1} w(k)+\bar{B}_{f} f(t), \\
r(t)=\bar{C} \xi(t)+\bar{D}_{d} d(t)+\bar{D}_{f} f(t)+\bar{L}_{2} w(t), \\
w(t)=\Delta z(t) \\
z(t)=\bar{H} \xi(t) \quad \Delta \in \Delta,
\end{gathered}
$$

where $\xi(t)=\left[x(t)^{T}, x_{f}(t)^{T}\right]^{T}$,

$$
\begin{gathered}
{\left[\begin{array}{c|c|c|c}
\bar{A} & \bar{B}_{d} & \bar{B}_{f} & \bar{L}_{1} \\
\hline \bar{C} & \bar{D}_{d} & \bar{D}_{f} & \bar{L}_{2}
\end{array}\right]} \\
\quad=\left[\begin{array}{cc|c|c|c}
A & 0 & B_{1} & B_{2} & L_{1} \\
B_{f} C & A_{f} & B_{f} D_{1} & B_{f} D_{2} & B_{f} L_{2} \\
\hline D_{f} C & C_{f} & D_{f} D_{1} & D_{f} D_{2} & D_{f} L_{2}
\end{array}\right] \\
\bar{H}=\left[\begin{array}{ll}
H & 0
\end{array}\right] .
\end{gathered}
$$

2.2. Problem Formulation. The design problem of the FD filter to be addressed in this paper can be expressed as follows.

The design objective: consider a class of uncertain linear systems (1); the FD filter (4) is designed such that the augmented model (5) is stable; the disturbance effects on the residual are minimized in both the fault-free case and the fault cases, while the fault effects on the residual are maximized in the fault cases. To detect actuator faults, our design objective of the FD filter can now be formulated as the following optimization problem:

$\max \beta$

$$
\text { s.t. } \delta_{\max }\left(G_{r d}(j \omega)\right)<\gamma, \quad \gamma>0, \forall|\omega| \leq \bar{\omega}_{1}, \Delta \in \Delta
$$

$$
\delta_{\min }\left(G_{r f}(j \omega)\right)>\beta \quad \beta>0, \forall|\omega| \leq \bar{\omega}_{2}, \Delta \in \Delta,
$$


where $\bar{\omega}_{1}$ is the upper bounds on the frequency for the disturbance and $\bar{\omega}_{2}$ is the upper bounds on the frequency for the fault. $G_{r d}$ is the transfer function from the disturbance $d$ to the residual signal $r$ with fault-free case and fault cases. $G_{r f}$ is the transfer function from the fault $f$ to the residual signal $r$ with fault cases.

Remark 2. Condition (8) describes the disturbance attenuation condition in both the fault-free case and fault cases. Condition (9) is formulated as the sensitiveness performance in the fault cases.

Before ending this section, the following lemmas and proposition shall be recalled to prove our main results for the FD filter design.

Lemma 3 (see [18]). Given a symmetric matrix $\Theta \in R^{n \times n}$ and two matrices $\mathscr{M}$ and $\mathscr{H}$ of column dimension $n$, there exists matrix $\mathscr{F}$ that satisfies $\Theta+\mathscr{M}^{T} \mathscr{F} \mathscr{H}+\mathscr{H}^{T} \mathscr{F}^{T} \mathscr{M}<0$ if and only if the following two conditions hold:

$$
\mathscr{N}_{\mathscr{M}}^{T} \Theta \mathcal{N}_{\mathscr{M}}<0 \quad \mathcal{N}_{\mathscr{H}} \Theta \mathcal{N}_{\mathscr{H}}^{T}<0,
$$

where $\mathcal{N}_{\mathscr{M}}$ and $\mathcal{N}_{\mathscr{H}}$ denote arbitrarily bases of null space of $\mathscr{M}$ and $\mathscr{H}$, respectively.

Lemma 4 (see [19]). Let $\xi \in R^{n}, \mathcal{N} \in R^{n \times n}$, and $\mathscr{M} \in$ $R^{n \times m}$ such that rank $(\mathcal{N})<n$. The following statements are equivalent:

(1) $\xi^{T} \mathscr{M} \xi<0$, for $\forall \xi \neq 0$, subject to $\mathscr{N} \xi=0$;

(2) $\mathscr{N}^{\perp T} \mathscr{M} \mathcal{N}^{\perp}<0$, where $\mathscr{N}^{\perp}$ is the kernel of $\mathcal{N}$;

(3) $\mathscr{M}-\varepsilon \mathscr{N}^{T} \mathcal{N}<0$, for some scalar $\varepsilon \in R$;

(4) $\mathscr{M}+X^{T} \mathscr{N}+\mathscr{N}^{T} X<0$, for some matrix $X \in R^{n \times m}$.

Proposition 5 (see [11]). Consider the uncertainty $\Delta$ with $\theta \in$ $u$ defined in (3), $\Gamma \in \Gamma$ is defined as

$$
\Gamma:=\left\{\Gamma=\left[\begin{array}{c}
\Delta \\
I
\end{array}\right] \mid \Delta \in \Delta\right\} .
$$

Restrict the parametrization of matrices $\Lambda$ defined as

$$
\Lambda:=\left\{\Lambda \mid \Lambda=\left[\begin{array}{ll}
\Lambda_{11} & \Lambda_{12} \\
\Lambda_{12}^{T} & \Lambda_{22}
\end{array}\right]\right\}
$$

with $\Lambda_{22}$ as a positive complex Hermitian matrix of dimension $n \times n$. Consider that $\Lambda_{11}=-\left(\Lambda_{22} \otimes(R / \mathscr{X})+\Lambda_{1}\right) \in R^{n_{\theta} n \times n_{\theta} n}$ and

$$
\Lambda_{12}=\left(\begin{array}{cccc}
0 & p_{12} & \cdots & p_{1 n} \\
-p_{12} & 0 & \cdots & p_{2 n} \\
\vdots & \ddots & \ddots & \vdots \\
-p_{1 \widehat{n}} & \cdots & \cdots & 0
\end{array}\right) \in R^{n_{\theta} n \times n}
$$

where $\mathscr{R}$ describes the form of the ellipsoid for uncertainty in (3), and the elements of this parametrization $\left(\Lambda_{a}, p_{i j}\right)$ can take any values provided. Consider that $\Lambda_{a} \in R^{n_{\theta} n \times n_{\theta} n}$ has the following structure that:

$$
\begin{gathered}
\Lambda_{1} \in \Lambda_{\mathbf{1}}:\left\{\left[\begin{array}{cccc}
0 & k_{12} & \cdots & k_{1 n} \\
-k_{12} & 0 & \cdots & \vdots \\
\vdots & \ddots & \ddots & k_{(n-1) n} \\
-k_{1 n} & \cdots & -k_{(n-1 n)} & 0
\end{array}\right]\right. \text { । } \\
\left.k_{i j}=-k_{i j}^{T} \in R^{n_{\theta} \times n_{\theta}}\right\}
\end{gathered}
$$

and $p_{i j} \in R^{n}, i=1, \ldots, n, j=1, \ldots, n$. For $\Lambda \in \Lambda, \Gamma^{T} \Lambda \Gamma>0$, $\forall \Gamma \in \Gamma$.

\section{Fault Detection Design}

In this section, Lemma 6 and Proposition 7 are first given, and inequality conditions for performance indices (8) and (9) are also given. Based on these conditions, an algorithm based on LMIs is presented.

Lemma 6 (see [16]). Consider the following uncertain system:

$$
\begin{gathered}
\dot{x}(t)=\left(A+B_{1} \Delta\left(I-D_{11} \Delta\right)^{-1} C_{1}\right) x(t)+B_{2} u(t), \\
y(t)=\left(C_{2}+D_{21} \Delta\left(I-D_{11} \Delta\right)^{-1} C_{1}\right) x(t)+D_{22} u(t),
\end{gathered}
$$

with $\Delta \in \Delta$. Let a real symmetric matrix $\Pi$ and a positive scalar $₫$ be given. The following robust finite-frequency condition

$$
\left[G_{1}(j \omega)^{T} I\right] \Pi\left[G_{1}(j \omega)^{T} I\right]^{T}<0 \quad|\omega| \leq \omega, \Delta \in \Delta
$$

holds if there exist real symmetric matrices $\mathscr{P}, \mathscr{Q}, \Phi \in \Phi$, and $\Lambda \in \Lambda$ such that

$$
\begin{gathered}
{\left[\begin{array}{cc}
\mathscr{A} & \mathscr{B} \\
\mathscr{I} & 0
\end{array}\right]^{T}\left[\begin{array}{cc}
-\mathscr{Q} & \mathscr{P} \\
\mathscr{P} & \mathscr{\omega}^{2} \mathscr{Q}
\end{array}\right]\left[\begin{array}{cc}
\mathscr{A} & \mathscr{B} \\
\mathscr{I} & 0
\end{array}\right]<\left[\begin{array}{l}
\mathscr{C}^{T} \\
\mathscr{D}^{T}
\end{array}\right] \Phi[\mathscr{C} \quad \mathscr{D}],} \\
\mathbb{Q} \geq \mathscr{C}_{1}^{T} \Lambda \mathscr{C}_{1},
\end{gathered}
$$

where

$$
\begin{gathered}
\Phi:=\left\{\left[\begin{array}{ll}
\Psi & 0 \\
0 & \Pi
\end{array}\right]:\left[\begin{array}{ll}
\Gamma & 0 \\
0 & \Gamma
\end{array}\right]^{T} \Psi\left[\begin{array}{ll}
\Gamma & 0 \\
0 & \Gamma
\end{array}\right] \leq 0, \forall \Gamma \in \Gamma\right\}, \\
{\left[\begin{array}{ll}
\mathscr{A} & \mathscr{B} \\
\mathscr{C}_{1} & \mathscr{D}_{1} \\
\mathscr{C}_{2} & \mathscr{D}_{2}
\end{array}\right]:=\left[\begin{array}{cc|cc}
A & B_{1} & B_{2} & 0 \\
0 & 0 & 0 & I \\
\hline 0 & I & 0 & 0 \\
C_{1} & D_{11} & 0 & 0 \\
\hline C_{2} & D_{21} & D_{22} & 0 \\
0 & 0 & I & 0
\end{array}\right]} \\
{\left[\begin{array}{ll}
\mathscr{C} & \mathscr{D}
\end{array}\right]:=\left[\begin{array}{l|l}
\mathscr{C}_{11} & \mathscr{D}_{11} \\
\hline \mathscr{C}_{2} & \mathscr{D}_{2}
\end{array}\right]=\left[\begin{array}{c|c|c}
\mathscr{C}_{1} & 0 \\
\mathscr{C}_{1} \mathscr{A} & \mathscr{C}_{1} \mathscr{B} \\
\hline \mathscr{C}_{2} & \mathscr{D}_{2}
\end{array}\right] .}
\end{gathered}
$$


Proposition 7. Consider the uncertainty $\Delta$ with $\theta \in \mathcal{U}$ defined in (3) and $\Gamma \in \Gamma$ defined in (11). Restrict the parametrization of matrices $\Psi$ defined as

$$
\Psi=\left[\begin{array}{c|c}
\Psi_{11} & \Psi_{12} \\
\hline \Psi_{12}^{T} & \Psi_{22}
\end{array}\right]=\left[\begin{array}{cc|cc}
\Psi_{111} & \Psi_{112} & \Psi_{121} & \Psi_{122} \\
\Psi_{112}^{T} & \Psi_{113} & \Psi_{122}^{T} & \Psi_{123} \\
\hline \Psi_{121}^{T} & \Psi_{122} & \Psi_{221} & \Psi_{222} \\
\Psi_{122}^{T} & \Psi_{123}^{T} & \Psi_{222}^{T} & \Psi_{223}
\end{array}\right],
$$

where $\left[\begin{array}{cc}\Psi_{113} & \Psi_{123} \\ * & \Psi_{223}\end{array}\right]$ are positive complex Hermitian matrices of dimension $2 n \times 2 n ; \Psi_{111}, \Psi_{121}$, and $\Psi_{221}$ of the dimension $n_{\theta} n \times$ $n_{\theta} n$ have the structure with $-\left(\Psi_{113} \otimes(R / \mathscr{X})+\Psi_{1}\right),-\left(\Psi_{123} \otimes\right.$ $\left.(R / \mathscr{X})+\Psi_{2}\right)$, and $-\left(\Psi_{223} \otimes(R / \mathscr{X})+\Psi_{3}\right)$, respectively. Moreover

$$
\Psi_{p 2}=\left(\begin{array}{cccc}
0 & p_{p 2} & \cdots & p_{p n} \\
-p_{p 2} & 0 & \cdots & p_{p n} \\
\vdots & \ddots & \ddots & \vdots \\
-p_{p \widehat{n}} & \cdots & \cdots & 0
\end{array}\right) \in R^{n_{\theta} n \times n}, \quad p=11,12,22
$$

where $\mathscr{R}$ describes the form of the ellipsoid for uncertainty in (3), and the elements of this parametrization $\left(\Psi_{1}, \Psi_{2}, \Psi_{3}\right.$, $\left.p_{1 i j}, p_{2 i j}, p_{3 i j}, i=1, \ldots, n, j=1, \ldots, n\right)$ can take any values provided. For $t=1,2,3, i=1, \ldots, n$, and $j=1, \ldots, n$, $\Psi_{t} \in R^{n_{\theta} n \times n_{\theta} n}$ has the following structure:

$$
\begin{gathered}
\Psi_{t} \in \Psi_{\mathbf{t}}:\left\{\left[\begin{array}{cccc}
0 & k_{t 12} & \cdots & k_{t 1 n} \\
-k_{t 12} & 0 & \cdots & \vdots \\
\vdots & \ddots & \ddots & k_{t(n-1) n} \\
-k_{t 1 n} & \cdots & -k_{t(n-1) n} & 0
\end{array}\right] \mid\right. \\
\left.k_{t i j}=-k_{t i j}^{T} \in R^{n_{\theta} \times n_{\theta}}\right\},
\end{gathered}
$$

and $p_{t i j} \in R^{n}$. Then, for $\Psi \in \Psi$, it has

$$
\left[\begin{array}{ll}
\Gamma & 0 \\
0 & \Gamma
\end{array}\right]^{T} \Psi\left[\begin{array}{ll}
\Gamma & 0 \\
0 & \Gamma
\end{array}\right]>0, \quad \forall \Gamma \in \Gamma,
$$

where $\Gamma$ is defined in (11).

Proof. Let all $\Psi_{p 2}, \Psi_{t}, p \in\{11,12,22\}, t \in\{1,2,3\}$ have the structure in the statement of the proposition:

$$
\Psi_{p 2}^{T} \Delta+\Delta^{T} \Psi_{p 2}=0, \quad \Delta^{T} \Psi_{t} \Delta=0 .
$$

Therefore, for every $\Psi \in \Psi$, we can have

$$
\begin{aligned}
{\left[\begin{array}{ll}
\Gamma & 0 \\
0 & \Gamma
\end{array}\right]^{T} \Psi\left[\begin{array}{ll}
\Gamma & 0 \\
0 & \Gamma
\end{array}\right] } & =\left[\begin{array}{cc}
\Delta & 0 \\
0 & I \\
\Delta & 0 \\
0 & I
\end{array}\right]^{T} \Psi\left[\begin{array}{cc}
\Delta & 0 \\
0 & I \\
\Delta & 0 \\
0 & I
\end{array}\right] \\
& =\left[\begin{array}{rr}
\Delta^{T} \Psi_{111} \Delta^{T}+\Psi_{112}^{T} \Delta+\Delta^{T} \Psi_{112}+\Psi_{113} & \Delta^{T} \Psi_{121} \Delta^{T}+\Psi_{122}^{T} \Delta+\Delta^{T} \Psi_{122}+\Psi_{123} \\
* & \Delta^{T} \Psi_{221} \Delta^{T}+\Psi_{222}^{T} \Delta+\Delta^{T} \Psi_{222}+\Psi_{223}
\end{array}\right] \\
& =\left[\begin{array}{rr}
\left(1-\theta^{T} \frac{R}{\mathscr{X}} \theta\right) \Psi_{111} & \left(1-\theta^{T} \frac{R}{\mathscr{X}} \theta\right) \Psi_{121} \\
* &
\end{array}\right] .
\end{aligned}
$$

When $\theta \in \mathcal{U}$ and $\left[\begin{array}{cc}\Psi_{113} & \Psi_{123} \\ * & \Psi_{223}\end{array}\right]>0$, we can have

$$
\left[\begin{array}{ll}
\Gamma & 0 \\
0 & \Gamma
\end{array}\right]^{T} \Psi\left[\begin{array}{ll}
\Gamma & 0 \\
0 & \Gamma
\end{array}\right]>0, \quad \forall \Gamma \in \Gamma .
$$

\subsection{The Disturbance Attenuation Condition}

Theorem 8. Consider the system in (5) with $\Delta \in \Delta$. A real symmetric matrix $\Pi_{a}=\left[\begin{array}{cc}1 & 0 \\ 0 & -\gamma^{2} I\end{array}\right]$ is given. Condition

$$
\delta_{\max }\left(G_{r d}(j \omega)\right)<\gamma, \quad|\omega|<\bar{\omega}_{2}, \Delta \in \Delta
$$

holds if there exist matrix variables $\widehat{A}, \widehat{B}, \widehat{C}, \widehat{D}, M, R, Y_{a}, E_{a}$, $L_{a}, N_{a}, W_{a}, S_{a}$,

$$
\begin{gathered}
P_{a}=\left[\begin{array}{lll}
P_{a 11} & P_{a 12} & P_{a 13} \\
P_{a 21} & P_{a 22} & P_{a 23} \\
P_{a 31} & P_{a 32} & P_{a 33}
\end{array}\right], \\
Q_{a}=\left[\begin{array}{lll}
Q_{a 11} & Q_{a 12} & Q_{a 13} \\
Q_{a 21} & Q_{a 22} & Q_{a 23} \\
Q_{a 31} & Q_{a 32} & Q_{a 33}
\end{array}\right],
\end{gathered}
$$




$$
\begin{gathered}
\Psi_{a}=\left[\begin{array}{cccc}
\Psi_{a 111} & \Psi_{a 112} & \Psi_{a 121} & \Psi_{a 122} \\
\Psi_{a 112}^{T} & \Psi_{a 113} & \Psi_{a 122^{T}} & \Psi_{a 123} \\
\Psi_{a 121}^{T} & \Psi_{a 122} & \Psi_{a 221} & \Psi_{a 222} \\
\Psi_{a 122}^{T} & \Psi_{a 123}^{T} & \Psi_{a 222}^{T} & \Psi_{a 223}
\end{array}\right], \\
\Lambda_{a}=\left[\begin{array}{ll}
\Lambda_{a 11} & \Lambda_{a 12} \\
\Lambda_{a 12}^{T} & \Lambda_{a 22}
\end{array}\right]
\end{gathered}
$$

of appropriate dimensions satisfying $Q_{a}>0, \Psi_{a} \in \Psi$, and $\Lambda_{a} \in \Lambda$ such that

$$
\left[\begin{array}{ccccccccc}
-Q_{a 11} & -Q_{a 12} & -Q_{a 13} & P_{a 11}-Y_{a}^{T} & P_{a 12}+E_{a}^{T} & P_{a 13}-L_{a}^{T} & a_{17} & a_{28} & 0 \\
* & -Q_{a 22} & -Q_{a 23} & P_{a 21}-M^{T} & P_{a 22}-M^{T} & P_{a 23} & a_{27} & a_{28} & 0 \\
* & * & -Q_{a 33} & Q_{a 31}-W_{a}^{T} & P_{a 32}-S_{a}^{T} & P_{a 33}-N_{a}^{T} & a_{37} & a_{38} & 0 \\
* & * & * & a_{44} & a_{45} & a_{46} & a_{47} & a_{48} & (\widehat{D} C)^{T} \\
* & * & * & * & a_{55} & a_{56} & a_{57} & a_{58} & \widehat{C}^{T} \\
* & * & * & * & * & a_{66} & a_{67} & a_{68} & \left(\widehat{D} L_{2}\right)^{T} \\
* & * & * & * & * & * & a_{77} & a_{78} & \left(\widehat{D} D_{1}\right)^{T} \\
* & * & * & * & * & * & * & a_{88} & 0 \\
* & * & * & * & * & * & * & * & I-H e(R)
\end{array}\right]<0
$$

where

$$
\begin{gathered}
a_{17}=-Y_{a}^{T} v_{1}, \quad a_{27}=-M^{T} v_{1}, \quad a_{37}=-W_{a}^{T} v_{1}, \\
a_{18}=-Y_{a}^{T} v_{2}, \quad a_{28}=-M^{T} v_{2}, \quad a_{38}=-W_{a}^{T} v_{2}, \\
a_{44}=\bar{\omega}_{1}^{2} Q_{a 11}+H^{T} \Psi_{a 113} H+A^{T} H^{T} \Psi_{a 223} H A \\
+\operatorname{He}\left(Y_{a} A+\widehat{B} C+H^{T} \Psi_{a 123} H A\right) \\
a_{45}=\bar{\omega}_{1}^{2} Q_{a 12}+\widehat{A}-A^{T} E_{a}^{T}+C^{T} \widehat{B}^{T}, \\
a_{46}=\bar{\omega}_{1}^{2} Q_{a 13}+H^{T} \Psi_{a 112}^{T}+A^{T} H^{T} \Psi_{a 122}^{T}+H^{T} \Psi_{a 123} H L_{1} \\
+A^{T} H^{T} \Psi_{a 223} H L_{1}+Y_{a} L_{1}+\widehat{B} L_{2}+A^{T} L_{a}^{T}, \\
a_{55}=\bar{\omega}_{1}^{2} Q_{a 22}+\operatorname{He}(\widehat{A}) \\
a_{56}=\bar{\omega}^{2}{ }_{1} Q_{a 23}-E_{a} L_{1}+\widehat{B} L_{2} \\
a_{66}^{2} Q_{a 33}+\Psi_{a 111}+L_{1}^{T} H^{T} \Psi_{a 223} H L_{1} \\
+H_{e}\left(\Psi_{a 122} H L_{1}+L_{a} L_{1}\right) \\
a_{48}=H^{T} \Psi_{a 122}^{T}+A^{T} H^{T} \Psi_{a 222}^{T}+W_{a}+\left(A^{T} Y_{a}^{T}+C^{T} \widehat{B}^{T}\right) v_{2}, \\
a_{57}=-E_{a} B_{1}+\widehat{B} D_{1}+\widehat{A}^{T} v_{1}, \\
a_{47}=H^{T} \Psi_{a 123} H B_{1}+A^{T} H^{T} \Psi_{a 223} H B_{1}+Y_{a} B_{1} \\
\left.+A_{1}^{T} Y_{a}^{T}+C^{T} \widehat{B}^{T}\right) v_{1},
\end{gathered}
$$

$$
a_{58}=S_{a}+\widehat{A}^{T} v_{2}
$$$$
a_{67}=\Psi_{a 122} H B_{1}+L_{1}^{T} H^{T} \Psi_{a 223} H B_{1}+L_{a} B_{1}
$$$$
+\left(L_{1}^{T} Y^{T}+L_{2}^{T} \widehat{B}^{T}\right) v_{1}
$$$$
a_{68}=\Psi_{a 121}+L_{1}^{T} H^{T} \Psi_{a 222}^{T}+N+\left(L_{1}^{T} Y_{a}^{T}+L_{2}^{T} \widehat{B}^{T}\right) v_{2},
$$$$
a_{77}=-\gamma^{2} I+B_{1}^{T} H^{T} \Psi_{a 223} H B_{1}
$$$$
+\operatorname{He}\left(\left(B_{1}^{T} Y_{a}^{T}+D_{1}^{T} \widehat{B}^{T}\right) v_{1}\right)
$$$$
a_{78}=B_{1}^{T} H^{T} \Psi_{a 222}^{T}+v_{1}^{T} W_{a}+\left(B_{1}^{T} Y_{a}^{T}+D_{1}^{T} \widehat{B}^{T}\right) v_{2},
$$$$
a_{88}=\Psi_{a 221}+\operatorname{He}\left(v_{2}^{T} W_{a}\right),
$$$$
\Lambda:=\left\{\Lambda_{a}: \text { satisfy (12) in Proposition 5 }\right\} \text {, }
$$$$
\Psi:=\left\{\Psi_{a}: \text { satisfy (20) in Proposition } 7\right\} \text {, }
$$

Proof. Substituting $\Pi_{a}$ into (17), $G_{r d}(j \omega)^{T} G_{r d}(j \omega)<\gamma^{2}$ which is equivalent to (27). By applying Lemma 6, (27) holds if

$$
\left[\begin{array}{cc}
\mathscr{A} & \mathscr{B}_{a} \\
\mathscr{I} & 0
\end{array}\right]^{T}\left[\begin{array}{cc}
-\mathscr{Q}_{a} & \mathscr{P}_{a} \\
\mathscr{P}_{a} & \bar{\omega}^{2} \mathscr{Q}_{a}
\end{array}\right]\left[\begin{array}{cc}
\mathscr{A} & \mathscr{B}_{a} \\
\mathscr{I} & 0
\end{array}\right]
$$




$$
\begin{gathered}
<\left[\begin{array}{l}
\mathscr{C}^{T} \\
\mathscr{D}^{T}
\end{array}\right]\left[\begin{array}{cc}
\Psi_{a} & 0 \\
0 & \Pi_{a}
\end{array}\right]\left[\begin{array}{ll}
\mathscr{C} & \mathscr{D}
\end{array}\right] \\
\mathscr{Q}_{a}>\mathscr{C}_{1}^{T} \Lambda_{a} \mathscr{C}_{1},
\end{gathered}
$$

where

$$
\begin{gathered}
{\left[\begin{array}{ll}
\mathscr{A} & \mathscr{B}_{a} \\
\mathscr{C}_{1} & \mathscr{D}_{1} \\
\mathscr{C}_{2} & \mathscr{D}_{2}
\end{array}\right]:=\left[\begin{array}{cc|cc}
\bar{A} & \bar{L}_{1} & \bar{B}_{d} & 0 \\
0 & 0 & 0 & I \\
\hline 0 & I & 0 & 0 \\
\bar{H} & 0 & 0 & 0 \\
\hline \bar{C} & \bar{L}_{2} & \bar{D}_{d} & 0 \\
0 & 0 & I & 0
\end{array}\right],} \\
{\left[\begin{array}{ll}
\mathscr{C} & \mathscr{D}
\end{array}\right]:=\left[\begin{array}{ll}
\mathscr{C}_{11} & \mathscr{D}_{11} \\
\hline \mathscr{C}_{2} & \mathscr{D}_{2}
\end{array}\right]=\left[\begin{array}{cc}
\mathscr{C}_{1} & 0 \\
\mathscr{C}_{1} \mathscr{A} & \mathscr{C}_{1} \mathscr{B}_{a} \\
\hline \mathscr{C}_{2} & \mathscr{D}_{2}
\end{array}\right] .}
\end{gathered}
$$

Let

$$
\Upsilon_{a}=\left[\begin{array}{ccc}
-\mathscr{Q}_{a} & \mathscr{P}_{a} & 0 \\
\mathscr{P}_{a} & \bar{\omega}_{2} \mathscr{Q}_{a}+\mathscr{C}_{11}^{T} \Psi_{a} \mathscr{C}_{11}+\mathscr{C}_{2}^{T} \Pi_{a} \mathscr{C}_{2} & \mathscr{C}_{11}^{T} \Psi_{a} \mathscr{D}_{11}+\mathscr{C}_{2}^{T} \Pi_{a} \mathscr{D}_{2} \\
0 & \mathscr{D}_{11}^{T} \Psi_{a} \mathscr{C}_{11}+\mathscr{D}_{2}^{T} \Pi_{a} \mathscr{C}_{2} & \mathscr{D}_{11}^{T} \Psi_{a} \mathscr{D}_{11}+\mathscr{D}_{2}^{T} \Pi_{a} \mathscr{D}_{2}
\end{array}\right]
$$

(32) is equivalent to

$$
\left[\begin{array}{cc}
\mathscr{A} & \mathscr{B}_{a} \\
I & 0 \\
0 & I
\end{array}\right]^{T} \Upsilon_{a}\left[\begin{array}{cc}
\mathscr{A} & \mathscr{B}_{a} \\
I & 0 \\
0 & I
\end{array}\right]<0
$$

And on the other hand,

$$
\left[\begin{array}{lll}
I & 0 & 0
\end{array}\right] \Upsilon_{a}\left[\begin{array}{lll}
I & 0 & 0
\end{array}\right]^{T}=-Q_{a}<0
$$

By combining (36) with (37), and applying Lemma 3, (32) holds if and only if

$$
\Upsilon_{a}+\left[\begin{array}{c}
-I \\
\mathscr{A}^{T} \\
\mathscr{B}_{a}^{T}
\end{array}\right] \mathscr{X}_{a}\left[\begin{array}{lll}
0 & I & 0 \\
0 & 0 & I
\end{array}\right]+\left[\begin{array}{lll}
0 & I & 0 \\
0 & 0 & I
\end{array}\right]^{T} \mathscr{X}_{a}^{T}\left[\begin{array}{c}
-I \\
\mathscr{A}^{T} \\
\mathscr{B}_{a}^{T}
\end{array}\right]^{T}<0
$$

where $\mathscr{X}_{a}$ introduced by Lemma 3 is the variable matrix of appropriate dimensions. Here, $\mathscr{X}_{a}$ is defined as $\mathscr{X}_{a}=$ $\left[\begin{array}{ll}x_{a 1} & x_{a 1} V_{1}\end{array}\right]$ and partition $\mathscr{X}_{a 1}$ into $\left[\begin{array}{ccc}x_{a 11} & -x_{a 12} & x_{a 13} \\ x_{21} & x_{22} & 0 \\ x_{a 31} & x_{a 32} & x_{a 33}\end{array}\right]$ where $x_{21}$ and $x_{22}$ are nonsingular matrices variable. $V_{1}$ is a given matrix, defined as $V_{1}=\left[\begin{array}{ccc}v_{1}^{T} & 0 & 0 \\ v_{2}^{T} & 0 & 0\end{array}\right]^{T}$.

Let $J=\operatorname{diag}\left\{I, x_{22}^{-1} x_{21}, I\right\}$, and define the linearizing change of the control variables as follows:

$$
\begin{gathered}
Q_{a}=J^{T} Q_{a} J, \\
X_{a 1}=\left[\begin{array}{ccc}
Y_{a}^{T} & -E_{a}^{T} & L_{a}^{T} \\
M^{T} & M^{T} & 0 \\
W_{a}^{T} & S_{a}^{T} & N_{a}^{T}
\end{array}\right] \\
=\left[\begin{array}{ccc}
x_{11} & -x_{12} x_{22}^{-1} x_{21} & x_{13} \\
x_{21}^{T} x_{22}^{-T} x_{21} & x_{21}^{T} x_{22}^{-T} x_{21} & 0 \\
x_{31} & x_{32} x_{22}^{-1} x_{21} & x_{33}
\end{array}\right], \\
P_{a}=J^{T} \mathscr{P}_{a} J,
\end{gathered}
$$

$$
\left[\begin{array}{cc}
\widehat{A}_{f} & \widehat{B}_{f} \\
\widehat{C}_{f} & 0
\end{array}\right]=\left[\begin{array}{cc}
x_{21}^{T} x_{22} & 0 \\
0 & I
\end{array}\right]\left[\begin{array}{cc}
A_{f} & B_{f} \\
C_{f} & 0
\end{array}\right]\left[\begin{array}{cc}
x_{22}^{T} x_{21} & 0 \\
0 & I
\end{array}\right]
$$

By Pre- and postmultiplying (38) by $\operatorname{diag}\left\{J^{T}, J^{T}, I\right\}$ and $\operatorname{diag}\{J, J, I\}$ and choos $\Psi_{a}$ satisfying Proposition 5, (38) is equivalent to

$$
\left[\begin{array}{ccc}
-Q_{a} & P_{a}-X_{a 1} & -X_{a 1} V_{1} \\
* & \bar{\omega}_{2} Q_{a}+\mathscr{C}_{11}^{T} \Psi_{a} \mathscr{C}_{11}+\mathscr{C}_{2}^{T} \Pi_{a} \mathscr{C}_{2}+\operatorname{He}\left(\mathscr{A}^{T} X_{a 1}\right) & \mathscr{C}_{11}^{T} \Psi_{a} \mathscr{D}_{11}+\mathscr{C}_{2}^{T} \Pi_{a} \mathscr{D}_{2}+\mathscr{A}^{T} X_{a 1} V_{1}+X_{a 1}^{T} \mathscr{B}_{a} \\
* & * & \mathscr{D}_{11}^{T} \Psi_{a} \mathscr{D}_{11}+\mathscr{D}_{2}^{T} \Pi_{a} \mathscr{D}_{2}+\operatorname{He}\left(\mathscr{B}_{a}^{T} X_{a 1} V_{1}\right)
\end{array}\right]<0
$$

where $\mathscr{A}, \mathscr{B}_{a}, \mathscr{C}_{11}, \mathscr{C}_{2}$ are the matrices which use the new defined variables $\widehat{A}_{f}, \widehat{B}_{f}, \widehat{C}_{f}$ in the matrices $\mathscr{A}, \mathscr{B}_{a}, \mathscr{C}_{11}, \mathscr{C}_{2}$.

By simple calculation, we can obtain that

$$
\begin{aligned}
& +\left[\begin{array}{c}
\mathfrak{S}^{T} \\
\bar{L}_{2}^{T} \\
\hline \bar{D}_{2}^{T} \\
0
\end{array}\right]\left[\begin{array}{lll}
\mathfrak{S} & \bar{L}_{2} \mid \bar{D}_{2} & 0
\end{array}\right] \\
& =\left[\begin{array}{c|c}
0 & 0 \\
\hline 0 & -\gamma^{2} \mathscr{I}_{0}
\end{array}\right] \\
& +\left[\frac{\mathfrak{E}_{1}^{T}}{\mathfrak{D}_{2}^{T}}\right]\left[\mathfrak{\mathfrak { V }}_{1} \mid \mathfrak{D}_{2}\right],
\end{aligned}
$$


where $\mathfrak{S}=\left[\begin{array}{ll}D_{f} C & \widehat{C}_{f}\end{array}\right], \mathfrak{C}_{1}=\left[\begin{array}{ll}\mathfrak{C} & \bar{L}_{2}\end{array}\right], \mathfrak{D}_{2}=\left[\begin{array}{ll}\bar{D}_{2} & 0\end{array}\right]$. By applying the Schur complement formula, the following can be obtained:

$$
\left[\begin{array}{cccc}
-Q_{a} & P_{a}-X_{a 1} & -X_{a 1} V_{1} & 0 \\
* & \bar{\omega}_{2} Q_{a}+\mathscr{C}_{11}^{T} \Psi_{a} \mathscr{C}_{11}+\operatorname{He}\left(\mathscr{A}^{T} X_{a 1}\right) & \mathscr{C}_{11}^{T} \Psi_{a} \mathscr{D}_{11}+\mathscr{A}^{T} X_{a 1} V_{1}+X_{a 1}^{T} \mathscr{B}_{a} & \mathfrak{C}_{1}^{T} \\
* & * & \mathscr{D}_{11}^{T} \Psi_{a} \mathscr{D}_{11}+\operatorname{He}\left(\mathscr{B}_{a}^{T} X_{a 1} V_{1}\right)-\gamma^{2} \mathscr{I}_{0} & \mathfrak{D}_{2}^{T} \\
* & * & * & -I
\end{array}\right]<0 .
$$

Then, pre-and postmultiply $\operatorname{diag}\left\{I, I, I, R^{T}\right\}$ to (42). Due to $-R^{T} R \leq I-\operatorname{He}(R)$ and defineing $\widehat{A}=M \widehat{A}_{f}, \widehat{B}=M \widehat{B}$, $\widehat{C}=R^{T} \widehat{C}_{f}, \widehat{D}=R^{T} D_{f},(29)$ hold. In addition, and pre- and postmultiplying (33) by $J_{1}^{T}$ and $J_{1}$, respectively, (33) becomes (30).

Hence, if conditions (29) and (30) holds, the augmented uncertain system (5) is stable and guarantees the $H_{\infty}$ performance (27), which completes the proof.

\subsection{The Fault Sensitiveness Condition for Faulty Case}

Theorem 9. Consider the system in (5) with $\Delta \in \Delta$. A real symmetric matrix $\Pi_{b}=\left[\begin{array}{cc}-1 & 0 \\ 0 & \beta^{2} I\end{array}\right]$. The following condition

$$
\delta_{\min }\left(G_{r f}(j \omega)\right)>\beta, \quad|\omega|<\bar{\omega}_{2}, \Delta \in \Delta
$$

holds if there exist matrix variables $\widehat{A}, \widehat{B}, \widehat{C}, \widehat{D}, M, R, Y_{b}, E_{b}$, $L_{b}, N_{b}, W_{b}, S_{b}$,

$$
\begin{gathered}
P_{b}=\left[\begin{array}{lll}
P_{b 11} & P_{b 12} & P_{b 13} \\
P_{b 21} & P_{b 22} & P_{b 23} \\
P_{b 31} & P_{b 32} & P_{b 33}
\end{array}\right], \\
Q_{b}=\left[\begin{array}{lll}
Q_{b 11} & Q_{b 12} & Q_{b 13} \\
Q_{b 21} & Q_{b 22} & Q_{b 23} \\
Q_{b 31} & Q_{b 32} & Q_{b 33}
\end{array}\right] \\
\Psi_{b}=\left[\begin{array}{llll}
\Psi_{b 111} & \Psi_{b 112} & \Psi_{b 121} & \Psi_{b 122} \\
\Psi_{b 112}^{T} & \Psi_{b 113} & \Psi_{b 122^{T}} & \Psi_{b 123} \\
\Psi_{b 121}^{T} & \Psi_{b 122} & \Psi_{b 221} & \Psi_{b 222} \\
\Psi_{b 122}^{T} & \Psi_{b 123}^{T} & \Psi_{b 222}^{T} & \Psi_{b 223}
\end{array}\right], \\
\Lambda_{b}=\left[\begin{array}{lll}
\Lambda_{b 11} & \Lambda_{b 12} \\
\Lambda_{b 12}^{T} & \Lambda_{b 22}
\end{array}\right]
\end{gathered}
$$

of appropriate dimensions satisfying $Q_{b}>0, \Psi_{b} \in \Psi$, and $\Lambda_{b} \in \Lambda$ such that

$$
\begin{aligned}
& {\left[\begin{array}{ccccccccc}
-Q_{b 11} & -Q_{b 12} & -Q_{b 13} & P_{b 11}-Y_{b}^{T} & P_{b 12}-E_{b}^{T} & P_{b 13}-L_{b}^{T} & -Y_{b}^{T} v_{3} & -Y_{b}^{T} v_{4} & 0 \\
* & -Q_{b 22} & -Q_{b 23} & P_{b 21}-M^{T} & P_{b 22}-M^{T} & P_{b 23} & -M^{T} v_{3} & -M^{T} v_{4} & 0 \\
* & * & -Q_{b 33} & P_{b 31}-W_{b}^{T} & P_{b 32}-S_{b}^{T} & P_{b 33}-N_{b}^{T} & -W_{b}^{T} v_{3} & -W_{b}^{T} v_{4} & 0 \\
* & * & * & c_{44} & b_{45} & b_{46} & b_{47} & b_{48} & -\mathscr{I}_{1} R^{T} \\
* & * & * & * & b_{55} & B_{56} & b_{57} & b_{58} & -\mathscr{I}_{1} R^{T} \\
* & * & * & * & * & b_{66} & b_{67} & b_{69} & -\mathscr{I}_{1} R^{T} \\
* & * & * & * & * & * & b_{77} & b_{78} & 0 \\
* & * & * & * & * & * & * & b_{88} & 0 \\
* & * & * & * & * & * & 0 & 0 & -I
\end{array}\right]<0,} \\
& {\left[\begin{array}{ccc}
Q_{b 11}-H^{T} \Lambda_{b 22} H & Q_{b 12} & Q_{b 13}-H^{T} \Lambda_{b 12}^{T} \\
* & Q_{b 22} & Q_{b 23} \\
* & * & Q_{b 33}-\Lambda_{b 11}
\end{array}\right]>0,}
\end{aligned}
$$

where

$$
\begin{aligned}
b_{44}= & \bar{\omega}_{2}^{2} Q_{b 11}+H^{T} \Psi_{113} H+A^{T} H^{T} \Psi_{b 223} H A \\
& +\mathrm{He}\left(Y_{b} A+\widehat{B} C+\mathscr{I}_{1} \widehat{D} C+H^{T} \Psi_{b 123} H A\right),
\end{aligned}
$$

$$
\begin{aligned}
b_{45}= & \bar{\omega}_{2}^{2} Q_{b 12}+\widehat{A}+A^{T} E_{b}^{T}+C^{T} \widehat{B}^{T}+C^{T} \widehat{B}^{T} \\
& +\left(\mathscr{I}_{1} \widehat{D} C\right)^{T}+\mathscr{I}_{1} \widehat{C} \\
b_{46}= & \bar{\omega}_{2}^{2} Q_{b 13}+H^{T} \Psi_{b 112}^{T}+A^{T} H^{T} \Psi_{b 122}^{T}
\end{aligned}
$$




$$
\begin{aligned}
& +H^{T} \Psi_{b 123} H L_{1}+A^{T} H^{T} \Psi_{b 223} H L_{1} \\
& +\widehat{B} L_{2}+A^{T} L_{b}^{T}+\mathscr{I}_{1} \widehat{D} L_{2}+C^{T} \widehat{D}^{T} \mathscr{J}_{1}^{T}, \\
& b_{55}=\bar{\omega}_{2}^{2} Q_{b 22}+\operatorname{He}\left(\widehat{A}+\mathscr{I}_{1} \widehat{C}\right) \text {, } \\
& b_{56}=\bar{\omega}_{2}^{2} Q_{b 23}+E L_{1}+\widehat{B} L_{2}+\mathscr{I}_{1} \widehat{D} L_{2}+\widehat{C}^{T} \mathscr{I}_{1}^{T} \text {, } \\
& b_{66}=\bar{\omega}_{2}^{2} Q_{b 33}+\Psi_{b 111}+L_{1}^{T} H^{T} \Psi_{b 223} H L_{1} \\
& +\mathrm{He}\left(\Psi_{b 122} H L_{1}+L_{b} L_{1}+\mathscr{I}_{1} \widehat{D} L_{2}\right) \text {, } \\
& b_{47}=H^{T} \Psi_{b 123} H B_{2}+A^{T} H^{T} \Psi_{b 223} H B_{2}+Y_{b} B_{2} \\
& +\widehat{B} D_{2}+\left(Y_{b} A+\widehat{B} C\right)^{T} v_{3}+\mathscr{I}_{1} \widehat{D} D_{2}, \\
& b_{48}=H^{T} \Psi_{b 122}^{T}+A^{T} H^{T} \Psi_{b 222}^{T}+W_{b}+\left(Y_{b} A+\widehat{B} C\right)^{T} v_{4} \text {, } \\
& b_{57}=E_{b} B_{2}+\widehat{B} D_{2}+\widehat{A}^{T} v_{3}+\mathscr{I}_{1} \widehat{D} D_{2}, \\
& b_{58}=S_{b}+\widehat{A}^{T} v_{4} \text {, } \\
& b_{67}=\Psi_{b 122} H B_{2}+L_{1}^{T} H^{T} \Psi_{b 223} H B_{2}+L_{b} B_{2} \\
& +\left(Y L_{1}+\widehat{B} L_{2}\right)^{T} v_{3}+\mathscr{I}_{1} \widehat{D} D_{2}, \\
& b_{68}=\Psi_{b 121}+L_{1}^{T} H^{T} \Psi_{b 222}^{T}+N_{b}+\left(Y_{b} L_{1}+\widehat{B} L_{2}\right)^{T} v_{4}, \\
& b_{77}=\beta^{2} I+B_{2}^{T} H^{T} \Psi_{a 223} H B_{2}+\operatorname{He}\left(\left(Y_{b} B_{2}+\widehat{B} D_{2}\right)^{T} v_{3}\right) \text {, } \\
& b_{78}=B_{2}^{T} H^{T} \Psi_{b 222}^{T}+v_{3}^{T} W_{b}+\left(Y_{b} B_{2}+\widehat{B} D_{2}\right)^{T} v_{4}, \\
& b_{88}=\Psi_{b 221}+\operatorname{He}\left(v_{4}^{T} W_{b}\right), \\
& \Lambda:=\left\{\Lambda_{b}: \text { satisfy (12) in Proposition } 5\right\} \text {, } \\
& \Psi:=\left\{\Psi_{b}: \text { satisfy (20) in Proposition } 7\right\},
\end{aligned}
$$

Proof. Substituting $\Pi_{b}$ into (17), $G_{r r_{0 i}}(j \omega)^{T} G_{r r_{0 i}}(j \omega)<\beta^{2} I$ which is equivalent to (43). Applying Lemma 6, (43) holds if

$$
\begin{gathered}
{\left[\begin{array}{cc}
\mathscr{A} & \mathscr{B}_{b} \\
\mathscr{I} & 0
\end{array}\right]^{T}\left[\begin{array}{cc}
-\mathscr{Q}_{b} & \mathscr{P}_{b} \\
\mathscr{P}_{b} & \bar{\omega}^{2} \mathscr{Q}_{b}
\end{array}\right]\left[\begin{array}{cc}
\mathscr{A} & \mathscr{B}_{b} \\
\mathscr{I} & 0
\end{array}\right]} \\
<\left[\begin{array}{c}
\mathscr{C}^{T} \\
\mathscr{D}^{T}
\end{array}\right]\left[\begin{array}{cc}
\Psi_{b} & 0 \\
0 & \Pi_{b}
\end{array}\right]\left[\begin{array}{ll}
\mathscr{C} & \mathscr{D}
\end{array}\right] \\
\mathscr{Q}_{b}>\mathscr{C}_{1}^{T} \Lambda_{b} \mathscr{C}_{1}
\end{gathered}
$$

hold.
Where

$$
\left[\begin{array}{cc}
\mathscr{A} & \mathscr{B}_{b} \\
\mathscr{C}_{1} & \mathscr{D}_{1} \\
\mathscr{C}_{2} & \mathscr{D}_{2}
\end{array}\right]:=\left[\begin{array}{cc|cc}
\bar{A} & \bar{L}_{1} & \bar{B}_{f} & 0 \\
0 & 0 & 0 & I \\
\hline 0 & I & 0 & 0 \\
\bar{H} & 0 & 0 & 0 \\
\hline \bar{C} & \bar{L}_{2} & \bar{D}_{f} & 0 \\
0 & 0 & I & 0
\end{array}\right]
$$

$$
\left[\begin{array}{ll}
\mathscr{C} & \mathscr{D}
\end{array}\right]:=\left[\begin{array}{c|c}
\mathscr{C}_{11} & \mathscr{D}_{11} \\
\hline \mathscr{C}_{2} & \mathscr{D}_{2}
\end{array}\right]=\left[\begin{array}{c|c}
\mathscr{C}_{1} & 0 \\
\mathscr{C}_{1} \mathscr{A} & \mathscr{C}_{1} \mathscr{B}_{b} \\
\hline \mathscr{C}_{2} & \mathscr{D}_{2}
\end{array}\right] .
$$

Let

$$
\Upsilon_{b}=\left[\begin{array}{ccc}
-\mathscr{Q}_{b} & \mathscr{P}_{b} & 0 \\
\mathscr{P}_{b} & \bar{\omega}_{2} \mathscr{Q}_{b}+\mathscr{C}_{11}^{T} \Psi_{b} \mathscr{C}_{11}+\mathscr{C}_{2}^{T} \Pi_{b} \mathscr{C}_{2} & \mathscr{C}_{11}^{T} \Psi_{b} \mathscr{D}_{11}+\mathscr{C}_{2}^{T} \Pi_{b} \mathscr{D}_{2} \\
0 & \mathscr{D}_{11}^{T} \Psi_{b} \mathscr{C}_{11}+\mathscr{D}_{2}^{T} \Pi_{b} \mathscr{C}_{2} & \mathscr{D}_{11}^{T} \Psi_{b} \mathscr{D}_{11}+\mathscr{D}_{2}^{T} \Pi_{b} \mathscr{D}_{2}
\end{array}\right]
$$

(48) is equivalent to

$$
\left[\begin{array}{cc}
\mathscr{A} & \mathscr{B}_{b} \\
I & 0 \\
0 & I
\end{array}\right]^{T} \Upsilon_{b}\left[\begin{array}{cc}
\mathscr{A} & \mathscr{B}_{b} \\
I & 0 \\
0 & I
\end{array}\right]<0
$$

and on the other hand,

$$
\left[\begin{array}{lll}
I & 0 & 0
\end{array}\right] \Upsilon_{b}\left[\begin{array}{lll}
I & 0 & 0
\end{array}\right]^{T}=-Q_{b}<0 .
$$

Combining (52) with (53) and applying Lemma 3, (48) holds if and only if

$$
\Upsilon_{b}+\left[\begin{array}{c}
-I \\
\mathscr{A}^{T} \\
\mathscr{B}_{b}^{T}
\end{array}\right] \mathscr{X}_{b}\left[\begin{array}{lll}
0 & I & 0 \\
0 & 0 & I
\end{array}\right]+\left[\begin{array}{lll}
0 & I & 0 \\
0 & 0 & I
\end{array}\right]^{T} \mathscr{X}_{b}^{T}\left[\begin{array}{c}
-I \\
\mathscr{A}^{T} \\
\mathscr{B}_{b}^{T}
\end{array}\right]^{T}<0,
$$

where $\mathscr{X}_{b}$ introduced by Lemma 3 is the variable matrix of appropriate dimensions. Here, $x_{b}$ is defined as $X_{b}=$ $\left[\begin{array}{ll}x_{b 1} & \mathscr{X}_{b 1} V_{2}\end{array}\right]$, where $\mathscr{X}_{b 1}=\left[\begin{array}{ccc}x_{b 11}-x_{b 12} & x_{b 13} \\ x_{21} & x_{22} & 0 \\ x_{b 31} & x_{b 32} & x_{b 33}\end{array}\right]$, where $x_{21}$ and $x_{22}$ are the same as in Theorem $8 . V_{2}$ is an given matrix, defined as $V_{2}=\left[\begin{array}{lll}v_{3}^{T} & 0 & 0 \\ v_{4}^{T} & 0 & 0\end{array}\right]^{T}$. Pre- and postmultiply (54) by $\operatorname{diag}\left\{J^{T}, J^{T}, I\right\}$ and $\operatorname{diag}\{J, J, I\}$, where $J$ is defined as Theorem 8 and choose $\Psi_{b}$ satisfying Proposition 5, (54) is equivalent to

$$
\left[\begin{array}{ccc}
-Q_{b} & P_{b}-X_{b 1} & -X_{b 1} V_{2} \\
* & \bar{\omega}_{2} Q_{b}-\mathscr{C}_{b 2}^{T} \mathscr{C}_{b 2}+\mathscr{C}_{11}^{T} \Psi_{b} \mathscr{C}_{11}+\operatorname{He}\left(X_{b 1}^{T} \overline{\mathscr{A}}_{b}\right) & -\mathscr{C}_{b 2}^{T} \mathscr{D}_{b 2}+\mathscr{C}_{11}^{T} \Psi_{b} \mathscr{D}_{11}+X_{b 1}^{T} \overline{\mathscr{B}}_{b}+\overline{\mathscr{A}}_{b} X_{b 1} V_{2} \\
* & * & -\mathscr{D}_{b 2}^{T} \mathscr{D}_{b 2}+\mathscr{D}_{11}^{T} \Psi_{b} \mathscr{D}_{11}+\beta^{2} \mathscr{F}_{0}+\operatorname{He}\left(\overline{\mathscr{B}}_{b}^{T} X_{b 1} V_{2}\right)
\end{array}\right]<0
$$


where

$$
\begin{aligned}
{\left[\begin{array}{cc}
\widehat{A}_{f} & \widehat{B}_{f} \\
\widehat{C}_{f} & 0
\end{array}\right]=\left[\begin{array}{cc}
x_{21}^{T} x_{22} & 0 \\
0 & I
\end{array}\right]\left[\begin{array}{cc}
A_{f} & B_{f} \\
C_{f} & 0
\end{array}\right]\left[\begin{array}{cc}
x_{22}^{T} x_{21} & 0 \\
0 & I
\end{array}\right], } \\
\mathscr{X}_{b 1}=J^{T} X_{1} J=\left[\begin{array}{ccc}
Y_{b}^{T} & E_{b}^{T} & L_{b}^{T} \\
M^{T} & M^{T} & 0 \\
W_{b}^{T} & S_{b}^{T} & N_{b}^{T}
\end{array}\right], \\
\overline{\mathscr{A}}_{b}=J^{-1} \mathscr{A} J=\left[\begin{array}{ccc}
A & 0 & L_{1} \\
\widehat{B}_{f} C & \widehat{A}_{f} & \widehat{B}_{f} L_{2} \\
0 & 0 & 0
\end{array}\right],
\end{aligned}
$$

$$
\begin{gathered}
\overline{\mathscr{B}}_{b}=J^{-1} \mathscr{B}_{b}=\left[\begin{array}{cc}
B_{2} & 0 \\
\widehat{B}_{f} D_{2} & 0 \\
0 & I
\end{array}\right], \\
\mathscr{C}_{b 2}=\left[\begin{array}{lll}
D_{f} C & \widehat{C}_{f} & D_{f} L_{2}
\end{array}\right], \quad \mathscr{D}_{b 2}=\left[\begin{array}{lll}
D_{f} D_{2} & 0
\end{array}\right],
\end{gathered}
$$

Condition (55) is nonlinear owing to the product terms $-C_{b 2}^{T} C_{b 2}$. To solve this problem, condition (55) is equivalent to

$$
\Xi_{b 2}^{T} \Theta \Xi_{b 2}<0
$$

where

$$
\begin{gathered}
\Xi_{b 2}=\left[\begin{array}{ccc}
I & 0 & 0 \\
0 & I & 0 \\
0 & 0 & I \\
0 & \mathscr{C}_{b 2} & \mathscr{D}_{b 2}
\end{array}\right], \\
\Theta=\left[\begin{array}{cccc}
-Q_{b} & P_{b}-X_{1} & -X_{1} V_{2} & 0 \\
* & \mathscr{C}_{11}^{T} \Psi_{b} \mathscr{C}_{11}+\operatorname{He}\left(X_{1}^{T} \overline{\mathscr{A}}_{b i}\right) & X_{1}^{T} \overline{\mathscr{B}}_{b}+\overline{\mathscr{A}}_{b i} X_{1} V_{2}+\mathscr{C}_{11}^{T} \Psi_{b} \mathscr{D}_{11} & 0 \\
* & * & \mathscr{D}_{11}^{T} \Psi_{b} \mathscr{D}_{11}+\beta^{2} \mathscr{I}_{0}+\operatorname{He}\left(\overline{\mathscr{B}}_{b}^{T} X_{b 1} V_{2}\right) & 0 \\
0 & 0 & 0 & -I
\end{array}\right] .
\end{gathered}
$$

Defining $G=\left[\begin{array}{llll}0 & \mathscr{C}_{b 2} & \mathscr{D}_{b 2} & -I\end{array}\right]$ satisfies $G \Xi_{b 2}=0$. Using Lemma 4 , and given a matrix $T$, (57) is equivalent to

$$
\Theta+T G+(T G)^{T}<0,
$$

where one notes $T$ as

$$
T=\left[\begin{array}{llll}
0 & \mathscr{R} & 0 & 0
\end{array}\right]^{T}, \quad \mathscr{R}=\left[\begin{array}{llll}
R \mathscr{F}_{1}^{T} & R \mathscr{F}_{1}^{T} & R \mathscr{F}_{1}^{T}
\end{array}\right] .
$$

Defining $\widehat{A}=M \widehat{A}_{f}, \widehat{B}=M \widehat{B}, \widehat{C}=R^{T} \widehat{C}_{f}$, and $\widehat{D}=R^{T} D_{f}$ and after some matrix manipulation, (59) becomes (45). By pre- and postmultiplying (49) by $J^{T}$ and $J$, respectively, (49) becomes (46).

Remark 10. Theorem 8 considers the attenuation performance for the disturbance $d(t)$, and the conservatism comes from the special structure of $\mathscr{X}_{a 1}$ and the same one in $\mathscr{X}_{a}$. Theorem 9 considers the sensitiveness performance for the fault $f(t)$, and the conservatism comes from the special structure of $\mathscr{X}_{b 1}$ and the same one in $X_{b}$. Then, the conservatism for Theorem 9 comes as the same as Theorem 8 . However, in order to obtain the better detection performance, the sensitiveness performance is considered over the conservatism.

3.3. Stability Condition for the Filter. Due to the fault tolerant controller, the closed-loop system is stable both in the faultfree cases and in the fault cases. Then, the condition that the augmented systems (1) is stable can translate into the condition that the FD filter is stable.
Lemma 11 (see [20]). By considering the FD filter (4), the FD filter is stable, if there exist matrix variables $M, \widehat{A}$ and $P_{s}>0$ satisfying

$$
\left[\begin{array}{ccc}
-\left(M+M^{T}\right) & \widehat{A}+P_{s} & M \\
* & -P_{s} & 0 \\
* & * & -P_{s}
\end{array}\right]<0 .
$$

3.4. Algorithm. In the precious sections, Theorems 8 and 9 and Lemma 11 have formulated the inequality conditions for the performance indexes (8) and (9) and the stable condition, respectively. Summarily, we have the following theorem.

Theorem 12. By considering the uncertain system model (1), there exists a FD filter (4) such that the augmented system model (5) is stable and satisfies the performance indices (8) and (9) if inequality conditions (29), (30), (45), (46), and (61) hold.

Proof. Combining Theorems 8-9 and Lemma 11, it is obviously that the theorem holds.

Remark 13. In Theorems 8-9, if the matrices $v_{i}, i \in\{1, \ldots, 4\}$ are given, all conditions (29), (30), (45), and (46) are the LMI conditions. Specially, if the disturbance and the fault are a scalar, respectively, $v_{i}$ is vector, and it can be easily chosen, which has been further confirmed in the simulink. In addition, Propositions 5 and 7 are used to directly deal with the ellipsoidal uncertainty; the variable structure is complex. However, all the variables in Propositions 5 and 7 is composed of linear matrices, and can be used for LMI conditions. 
The gain matrices $A_{f}, B_{f}, C_{f}$, and $D_{f}$ defining the FD filter can be derived by the means of a standard procedure. Denote $\widehat{A}, \widehat{B}, \widehat{C}, \widehat{D}$, and $M$ is the optimal solution of (7) with condition (29), (30), (45), (46), and (61):

(1) compute the $x_{21}, x_{22}$ by solving the following factorization problem $M=x_{21}^{T} x_{22}^{-1} x_{21}$;

(2) compute $\widehat{A}_{f}, \widehat{B}_{f}$ by $\widehat{A}, \widehat{B}$ and $M$. Compute $\widehat{C}_{f}, D_{f}$ by $\widehat{C}, \widehat{D}$ and $R$;

(3) finally, obtain the gain matrices of the FD filter

$$
\left[\begin{array}{cc}
\widehat{A}_{f} & \widehat{B}_{f} \\
\widehat{C}_{f} & 0
\end{array}\right]=\left[\begin{array}{cc}
x_{21}^{T} x_{22} & 0 \\
0 & I
\end{array}\right]\left[\begin{array}{cc}
A_{f} & B_{f} \\
C_{f} & 0
\end{array}\right]\left[\begin{array}{cc}
x_{22}^{T} x_{21} & 0 \\
0 & I
\end{array}\right]
$$

\section{Thresholds Computation}

After the parameter matrices of the FD filter $A_{f}, B_{f}, C_{f}$, and $D_{f}$ are designed, similar to that proposed in [21], the residual evaluation function $J_{r}(t)$ can be selected as

$$
J_{r}(t)=\sqrt{\frac{1}{t} \int_{0}^{t} r^{T}(\tau) r(\tau) d \tau .}
$$

Under fault-free condition, the residual $r(t)$ becomes

$$
r(t)=G_{r d}(j \omega) d(t),
$$

and namely, the Parseval Theorem (see [22]), one has that

$$
J_{r}(t) \leq\left\|G_{r d_{0}}\right\|_{\infty}\|d\|_{\mathrm{rms}} \leq\left\|G_{r d_{0}}\right\|_{\infty} \bar{d},
$$

where $\bar{d}$ is the upper bound on the disturbance. It is assumed that $\bar{d}$ is the known. As a consequence, the following threshold results:

$$
J_{\text {th }}=\left\|G_{r d_{0}}\right\|_{\infty} \bar{d}
$$

Based on this, the occurrence of faults can be detected by the following logic rule.

$$
\begin{gathered}
\left\|J_{r}\right\| \leq J_{\text {th }}, \quad \text { no alarm, } \\
\left\|J_{r}\right\|>J_{\text {th }}, \quad \text { with alarm. }
\end{gathered}
$$

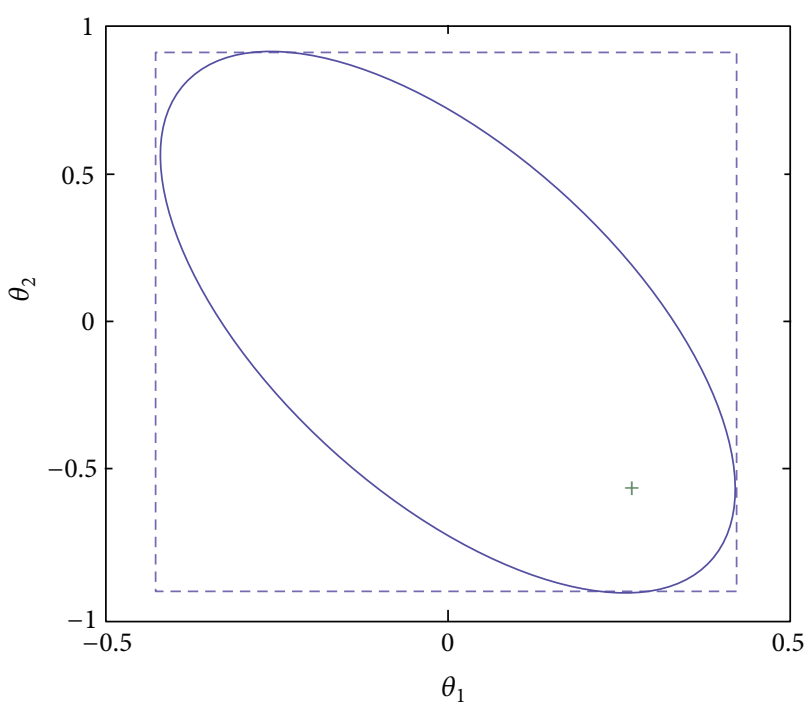

FIGURE 1: Ellipsoidal uncertainty.

\section{Example}

In this section, an example is given to illustrate the effectiveness of our design method. Consider the uncertain linear system by

$$
\begin{aligned}
& A=\left[\begin{array}{cc}
0.5 & 1 \\
-3 & -3
\end{array}\right], \quad B_{1}=\left[\begin{array}{c}
0.4 \\
-0.5
\end{array}\right], \quad B_{2}=\left[\begin{array}{c}
0.5 \\
-1
\end{array}\right], \\
& L_{1}=\left[\begin{array}{cccc}
0.2 & 0 & 0.1 & 0 \\
0 & 0 & 0 & 0.2
\end{array}\right], \quad C=\left[\begin{array}{ll}
1 & 1
\end{array}\right], \\
& D_{1}=0.5, \quad D_{2}=0.5, \\
& L_{2}=\left[\begin{array}{llll}
0 & 0 & 0 & 0
\end{array}\right], \quad H=\left[\begin{array}{ll}
1 & 0 \\
0 & 1
\end{array}\right] \text {, } \\
& \Delta=\left[\begin{array}{ll}
\theta & 0 \\
0 & \theta
\end{array}\right], \quad \theta=\left[\begin{array}{l}
\theta_{1} \\
\theta_{2}
\end{array}\right],
\end{aligned}
$$

and the uncertain parameters $\left[\begin{array}{c}\theta_{1}^{T} \\ \theta_{2}^{T}\end{array}\right] \in \mathscr{U}$ satisfy $\left\{\theta \mid \theta^{T} \mathscr{R} \theta<\right.$ $1\}$, as shown in Figure 1, where $\mathscr{R}=\left[\begin{array}{ll}1.25 & 0.25 \\ 0.25 & 1.25\end{array}\right]$. It is worth pointing out that if the method in this paper is adopted, the uncertain region is only in the ellipsoid (solid lines). Otherwise, the ellipsoid has to translate into the convex bounded polyhedral domain (dashed line) for the existing method to deal with the uncertainty, which enlarges the uncertain region and results in increasing the conservatism.

Given the matrices

$$
\begin{gathered}
v_{1}=\left[\begin{array}{c}
0 \\
-0.2
\end{array}\right], \quad v_{2}=\left[\begin{array}{llll}
0 & 0 & 0 & 0 \\
0 & 0 & 0 & 0
\end{array}\right], \\
v_{3}=\left[\begin{array}{l}
-0.5 \\
-0.5
\end{array}\right], \quad v_{4}=\left[\begin{array}{cccc}
1.5 & 1.5 & 1.5 & -1.5 \\
1 & 1 & -1 & -1
\end{array}\right],
\end{gathered}
$$

the disturbance is $d(t)=0.8 \sin (0.1 t) \exp (-0.05 t)$. Then, the upper bounds on the frequency for the disturbance and the reference input can choose $\bar{\omega}_{1}=0.5$ and $\bar{\omega}_{2}=0$, respectively. 


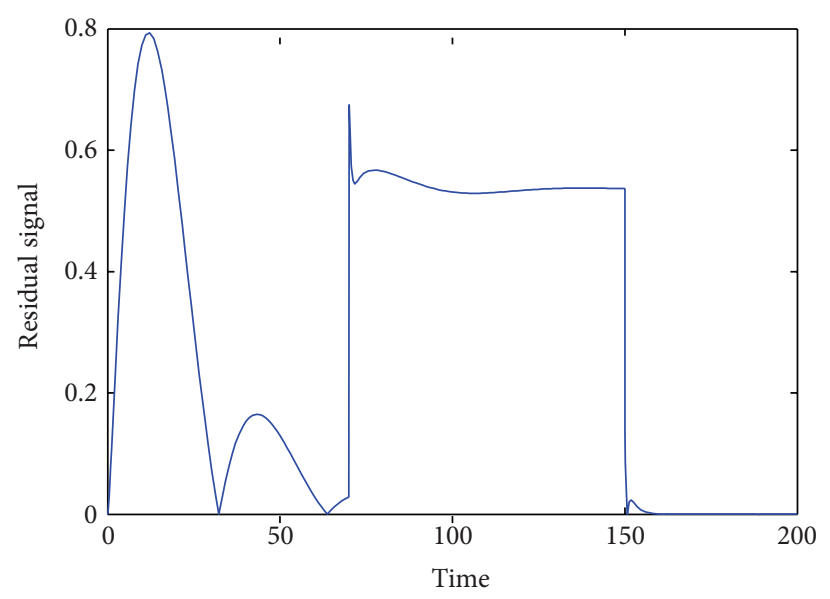

(a)

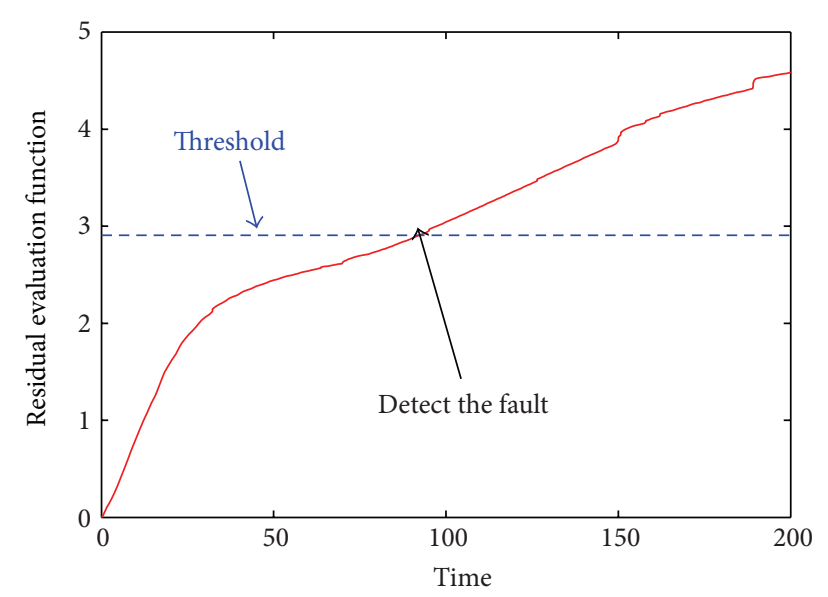

(b)

FIGURE 2: The residual signal and the residual evaluation function $J_{r}$.

Given $\gamma=1$, for the practical needs, we obtain the sensitivity performance $\beta=0.4234$ by solving the optimization problem (16) with the conditions in Theorem 12 and the FD filter with gain matrices as

$$
\begin{array}{ll}
A_{f}=\left[\begin{array}{cc}
-3.5304 & 0.0850 \\
-6.0843 & -0.2812
\end{array}\right], & B_{f}=\left[\begin{array}{l}
0.0696 \\
2.5117
\end{array}\right], \\
C_{f}=\left[\begin{array}{ll}
-0.0539 & -0.2526
\end{array}\right], & D_{f}=-1.3339 .
\end{array}
$$

Choose $\theta_{1}=0.2$ and $\theta_{1}=-0.6$. Considering the case that the fault occurs between $70 \mathrm{sec}$ and $150 \mathrm{sec}$, the residual signal and the residual evaluation function $J_{r}(t)$ and the threshold $J_{\text {th }}(t)$ are shown in Figure 2. Consider that $J_{r}(t)>J_{\text {th }}(t)$ at $92.5 \mathrm{sec}$, which means that the fault alarms when the outage fault occurs. Hence, the fault can be detected.

\section{Conclusions}

In this paper, the design problem of the FD filter for uncertain linear systems has been addressed. A method for the design problem is given in terms of the linear matrix inequality (LMI) optimization techniques. The new proposed method has two main advantages. The first one is to resolve the problem of designing FD filters subjected to the model with ellipsoidal parametric uncertainty region, while this problem cannot be directly resolved using the existing method. The second one is that the finite-frequency performances of the fault and the disturbance have been detected.

\section{References}

[1] J. Chen and P. R. Patton, Robust Model-Based Fault Diagnosis For Dynamic Systems, Kluwer Academic Publishers, Boston, Mass, USA.

[2] T. Li, L. Guo, and L. Y. Wu, "Observer-based optimal fault detection using PDFs for time-delay stochastic systems," Nonlinear Analysis: Real World Applications, vol. 9, no. 5, pp. 2337-2349, 2008.
[3] I. Hwang, S. Kim, Y. Kim, and C. E. Seah, "A survey of fault detection, isolation, and reconfiguration methods," IEEE Transactions on Control Systems Technology, vol. 18, no. 3, pp. 636-653, 2010.

[4] E. G. Nobrega,, M. O. Abdalla, and K. M. Grigoriadis, "LMIbased filter design for fault detection and isolation," in Proceedings of the 39th IEEE Confernce on Decision and Control (CDC '00), pp. 4329-4334, Sydney, Australia, December 2000.

[5] T. Li and L. Guo, "Optimal fault-detection filtering for nonGaussian systems via output PDFs," IEEE Transactions on Systems, Man, and Cybernetics A, vol. 39, no. 2, pp. 476-481, 2009.

[6] W. Li, F. Jiang, Z. Wang, G. Zhou, and Z. Zhu, "Fault detection of Markov jumping linear systems," Mathematical Problems in Engineering, vol. 2012, Article ID 141867, 27 pages, 2012.

[7] M. Hou and R. J. Patton, "A LMI approach to $H_{-} / H \infty$ fault detection observers," in Proceedings of the UKACC International Conference on Control, pp. 305-310, London, UK, September 1996.

[8] I. M. Jaimoukha, Z. Li, and V. Papakos, "A matrix factorization solution to the $H_{-} / H \infty$ fault detection problem," Automatica, vol. 42, no. 11, pp. 1907-1912, 2006.

[9] X. Li and H. H. T. Liu, "Minimum system sensitivity study of linear discrete time systems for fault detection," Mathematical Problems in Engineering, vol. 2013, Article ID 276987, 13 pages, 2013.

[10] X. J. Cai and F. Wu, "Robust fault detection and isolation for parameter-dependent LFT systems," International Journal of Robust and Nonlinear Control, vol. 20, no. 7, pp. 764-776, 2010.

[11] M. Barenthin, X. Bombois, H. Hjalmarsson, and G. Scorletti, "Identification for control of multivariable systems: controller validation and experiment design via LMIs," Automatica, vol. 44, no. 12, pp. 3070-3078, 2008.

[12] R. D. Braatz and O. D. Crisalle, "Robustness analysis for systems with ellipsoidal uncertainty," International Journal of Robust and Nonlinear Control, vol. 8, no. 13, pp. 1113-1117, 1998.

[13] X. Bombois, M. Gevers, G. Scorletti, and B. D. O. Anderson, "Robustness analysis tools for an uncertainty set obtained by prediction error identification," Automatica, vol. 37, no. 10, pp. 1629-1636, 2001. 
[14] X. Bombois, H. Hjalmarsson, and G. Scorletti, "Identification for robust $\mathrm{H}_{2}$ deconvolution filtering," Automatica, vol. 46, no. 3, pp. 577-584, 2010.

[15] A. Sadeghzadeh and H. Momeni, "Fixed-order robust $H_{\infty}$ control and control-oriented uncertainty set shaping for systems with ellipsoidal parametric uncertainty," International Journal of Robust and Nonlinear Control, vol. 21, no. 6, pp. 648-665, 2011.

[16] G.-H. Yang and H. Wang, "Fault detection for a class of uncertain state-feedback control systems," IEEE Transactions on Control Systems Technology, vol. 18, no. 1, pp. 201-212, 2010.

[17] T. Iwasaki, S. Hara, and H. Yamauchi, "Dynamical system design from a control perspective: finite frequency positiverealness approach," IEEE Transactions on Automatic Control, vol. 48, no. 8, pp. 1337-1354, 2003.

[18] X. J. Li and G. H. Yang, "Fault detection filter design for stochastic time-delay systems with sensor faults," International Journal of Systems Science, vol. 43, no. 8, 2012.

[19] K. Hu and J. Yuan, "Improved robust $H_{\infty}$ filtering for uncertain discrete-time switched systems," IET Control Theory \& Applications, vol. 3, no. 3, pp. 315-324, 2009.

[20] X. J. Li, Research on robust adaptive fault detection and isolation approaches [Ph.D. thesis], Northeastern University, Liaoning, China.

[21] P. M. Frank and X. Ding, "Frequency domain approach to optimally robust residual generation and evaluation for modelbased fault diagnosis," Automatica, vol. 30, no. 5, pp. 789-804, 1994.

[22] S. Boyd and C. Barratt, Linear Controller Design: Limites of Performance, Prentice Hall, Englewood Cliffs, NJ, USA, 1991. 


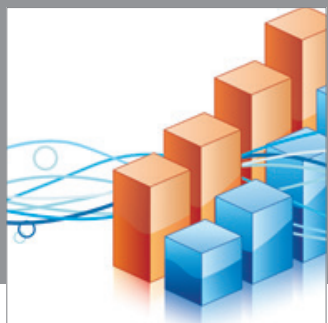

Advances in

Operations Research

mansans

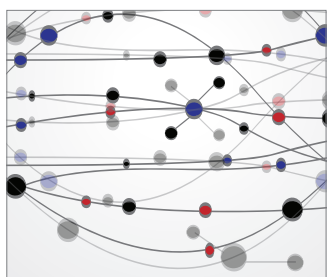

The Scientific World Journal
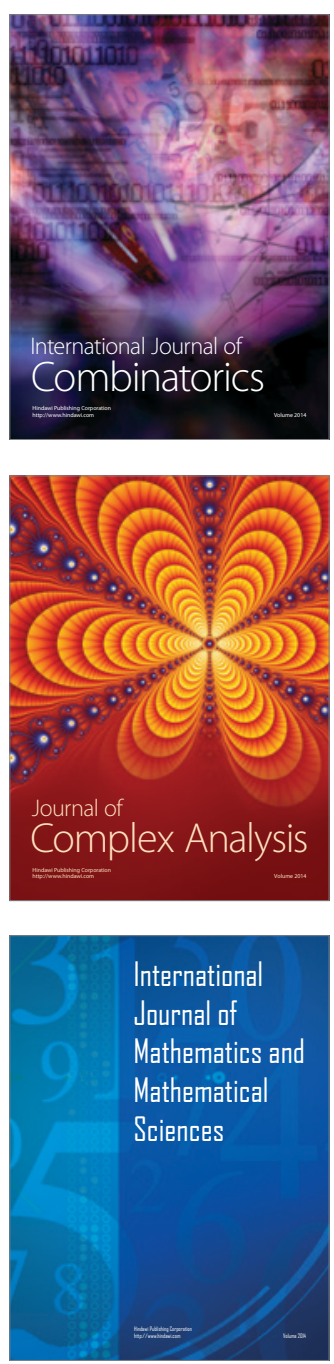
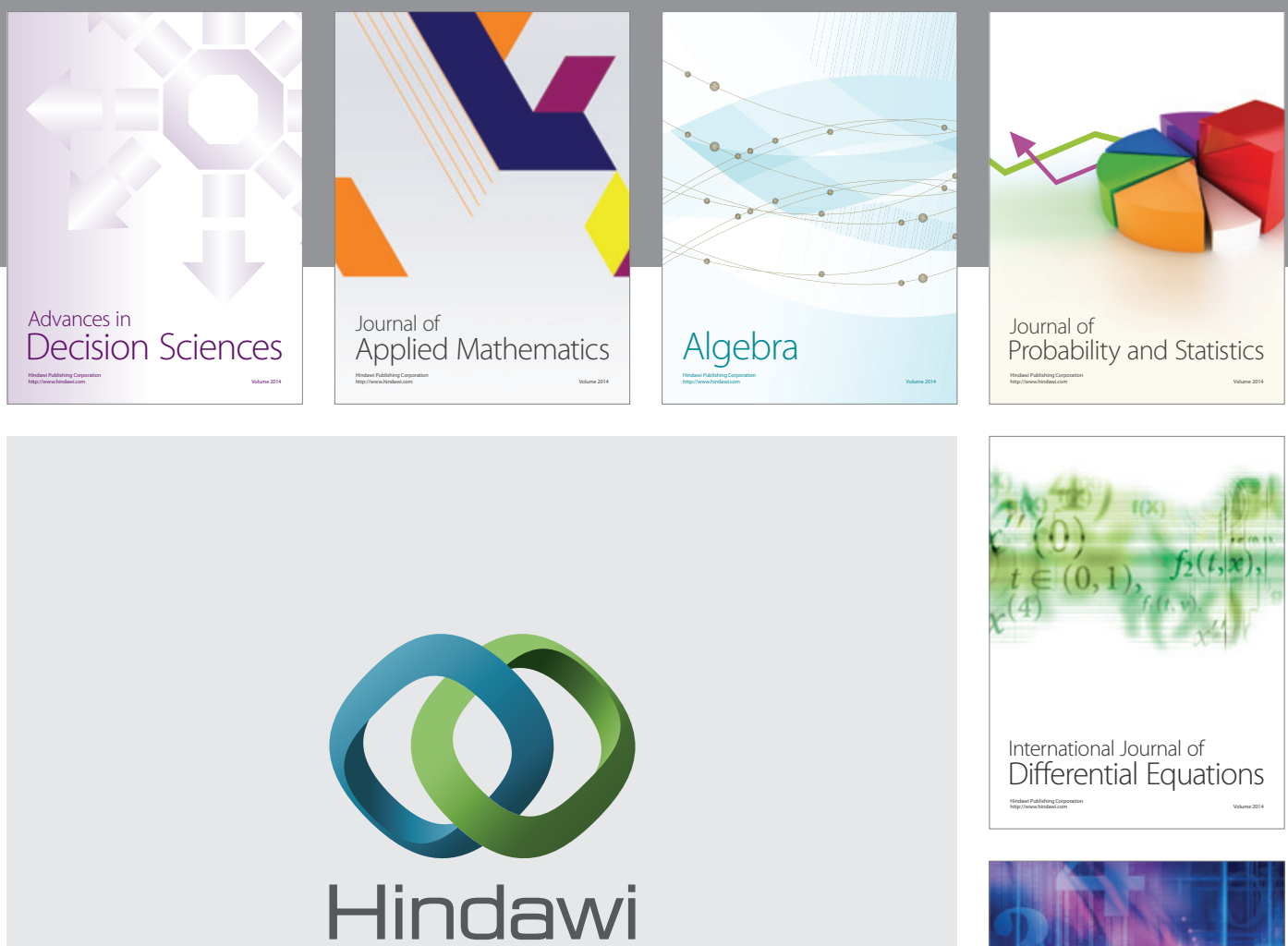

Submit your manuscripts at http://www.hindawi.com
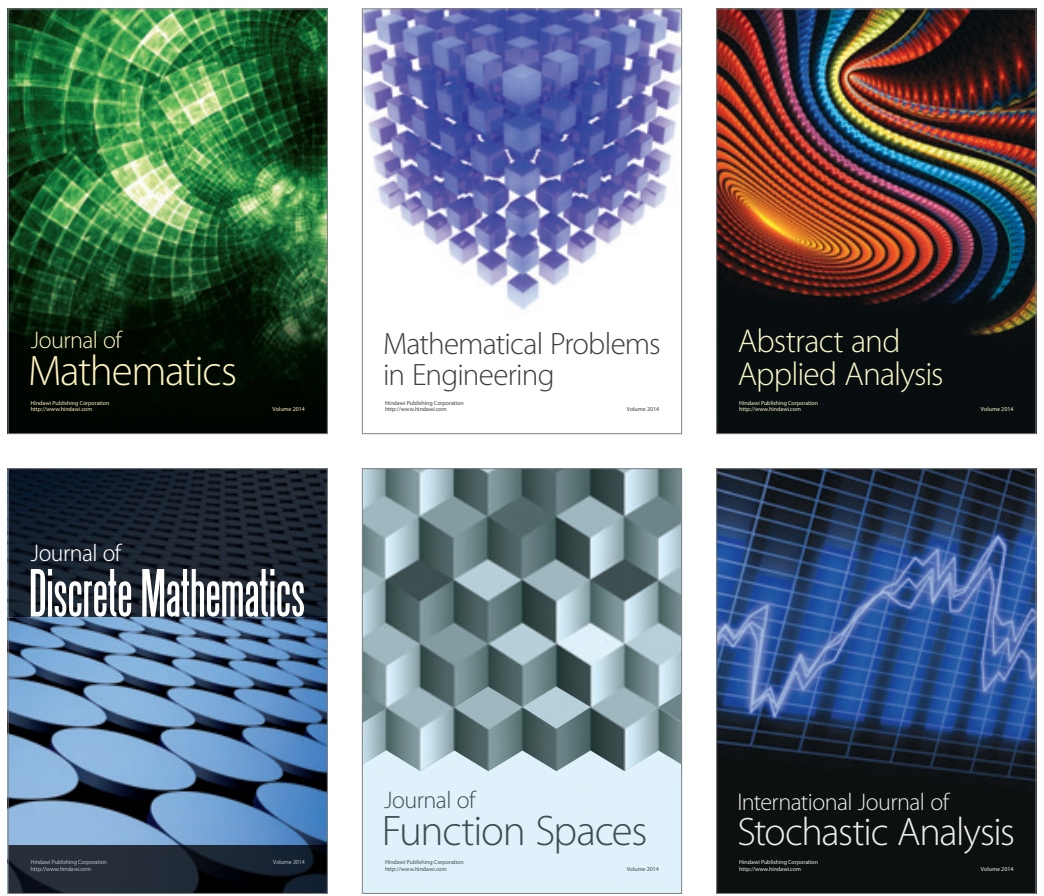

Journal of

Function Spaces

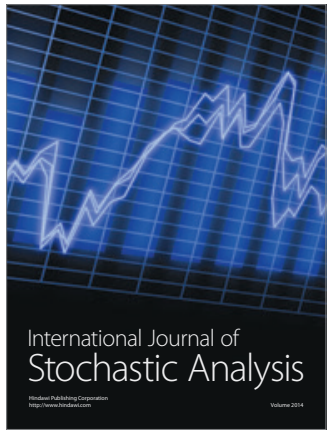

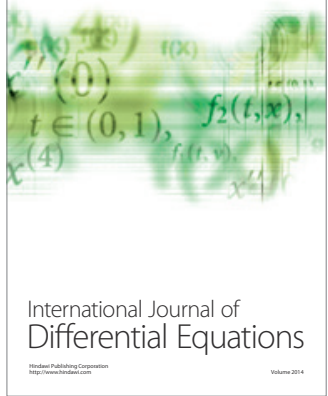
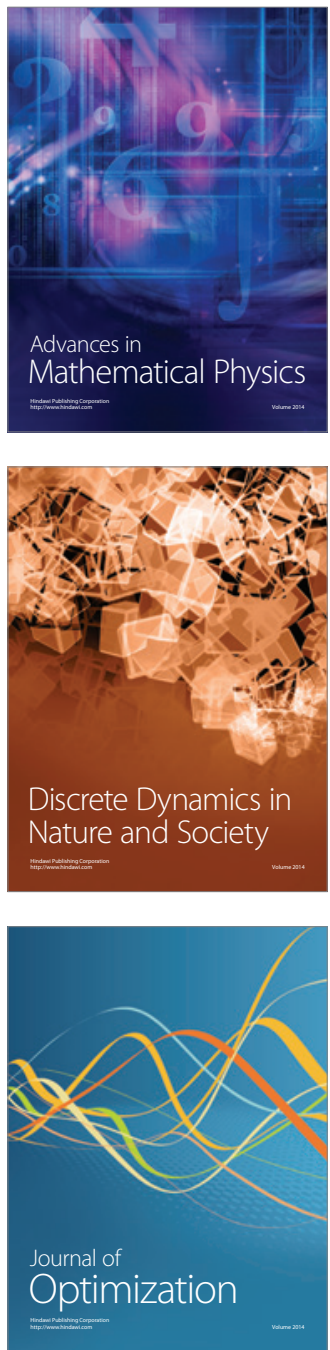